\title{
PRODUCTION DATA ACQUISITION AND ANALYSIS MANAGEMENT SYSTEM: AN EXAMPLE BASED ON A STUDY OF AUTOMOTIVE SUPPLIER SOLUTION
}

\author{
DOI: 10.12776/QIP.V17I2.260
}

\section{LUBOMIR LENGYEL}

\section{INTRODUCTION}

During last decade there has been a significant change in driving the production facilities. Major focus of companies is now placed on lean manufacturing, improving of efficiency and productivity of operations to maximize output and in the same time minimizing required resources.

This change is as well affecting the data collection. For the efficient and quick decision making process is mandatory to have reliable, qualitatively accurate and real time available information. Majority of the corporations and now as well mid-size companies invested already into the ERP systems allowing them to have a really detailed overview about production capabilities. However all those systems are working only in case of availability of data. Data can be extracted either automatically or manually and this process has to take place in the area of occurrence of information source. In industrial production plants automatisation is on the rise, however there are still plenty of the source locations where only manual entry of data by operator is possible.

Quality of data coming from manual entry of information is a key element in efficiency of decision making process for all support functions and as well management allowing them to quickly react on changing circumstances of working environment.

This paper is intended to quality organization members so as other broad audience dealing with manual entry issues and analysis of such acquired.

\section{METHODOLOGY AND ANALYSIS}

Quality of data coming from manual entry of informations is a key element in efficiency of decision making process for all support functions and as well management allowing them to quickly react on changing circumstances of working environment. 
Major issues arising from manual entry of data can be summarized according Warren Wolfe (Points North, 2013) to delayed cost reporting, slowed decision making process, inaccurate reporting due to manual errors, labour involved with documentation storage.

Concerning point of costs we can assume as well costs of labour needed to perform manual data entry and the work time of the operators/supervisors needed to collect information and have in hand written for typing into the system.

Solution for above mentioned issues is the automated data acquisition process using specialized system for manual data entry placed in the area of data occurrence enabling fast data input.

Majority of currently available systems are based on web client architecture and are using possibilities of the web based forms and checklists e.g. ProductionACE report generator (Production Process, 2013).

This solution is cheap, easy to use, adjustable and accessible for wide population. However it is very similar to manual data typing tools and in the end is not preventing an operator to have mistyping defects. Reliability and stability of system in case of big data loads (more than 10,000 records per hour) can be also questionable.

Other solutions are based on the special applications running on the workstations available directly in production able to directly communicate with the superior systems like ERP's, e.g. SuiteMDI ${ }^{\mathrm{TM}}$ (CADCAM Integration, 2013). Those software are allowing to improve the manual data acquisition by implementing of checking algorithms and cooperation with superior ERP's. They are as well much more stable and reliable in difficult production environment.

Another possibilities are specialized software's and modules running as a part of the ERP systems like Pegasie IDEA (Pegasie Technologies, 2013). This solution is the highly efficient, reliable, and stable. On the other hand as well most costly possibility of solving manual entry issues.

Overall all of those systems are lacking one of the most important features and are not taking into account one of the major principles of getting data based on the philosophy WYSIWYG - what you see is what you get.

Operator performing a data entry is required to select the proper data group by selecting from written possibilities. Operator is as well required to input the bulk data using a keyboard. Operator is requested to confirm the data by clicking on the buttons using a mouse or any other I/O device. All of those requirements are then increasing a time needed to perform a data entry operation, needs further investments into the hardware, requires a space where to locate them, are not following WYSIWYG principle and in the end are not so quick as required and with certain amount of human errors data quality is still not as good as required (Robbins, 2013). 


\section{CASE STUDY: PRODUCTION DATA ACQUISITION AND ANALYSIS MANAGEMENT SYSTEM IN FAURECIA KOSICE}

Faurecia Slovakia, plant Kosice is a part of the company Faurecia which is leading supplier of automotive industry worldwide. Plant Kosice is a part of the Faurecia's business group Interior Systems and is specializing in the leather processing. Kosice plant is delivering Instrument Panels, Door Panels and various interior part of the cars covered with natural or artificial leather to 11 customers in Germany, France, Great Britain, South Africa, Poland and Slovakia. OEM's supplied by Kosice are Daimler, Land Rover, PSA, Ford, and Volvo.

In plant Faurecia Kosice various covering techniques are used for covering of the interior systems product groups. Automatic covering for simple plain surfaces like central console covers, semiautomatic covering using presses with specialized tools for more difficult products like parts of doorpanels up to the fully manual covering of difficult shaped products like instrument panels. All of those processes are still requiring certain operator involvement for loading and unloading of material finished products into the machine and finalizing the part. The most important point of aspect control in the end of the production line is performed on every of the part being produced and of course have to be performed by human as the assessment of the whole product depends on many factors. Surface cover (leather or artificial leather) natural defects (weins, scars, wrinkles) have to be assessed according agreed standards with OEM, various levels of aspect defects can be accepted due to inhomogeneity of the materials etc.

Result of this final check is than the most important information coming out of the production line as all other information about product functional characteristics are recorded automatically or machine based data are taken into account.

Originally all of those information has been recorded on paper checklists which were in the end of the shift collated together by gapleaders and supervisors were manually typing them into the ERP system and as well to MS Excel to allow further possibilities of reporting and analysis. This kind of process is very common in majority of industrial productions as requires 0 investments in the time of implementation as time needed for this manual entry is included in the cycle time of the final control operation.

Due to the nature of above mentioned solution there are obvious typing defects where operator wrongly records number of items declared as NOK, will possibly mix up the number in reference codes etc. Secondly during typing of data by supervisor additional data noise can be experienced by performing same mistakes.

Another difficulty is the availability of such gathered data. As the information are available only in the end of the production shift, there is a minimum 10 hours delay (normally 24 hours) between data occurrence and data availability. 
Analysis of such acquired data can be performed only after mentioned 24 hours introducing a significant delay in decision making process what can be critical in case of significant deviation from standard. To decrease such risks, various solutions including escalation criteria based again on human factor are implemented, introducing further unclarities and information noises into the process.

Due to the nature of such acquired data (numerical and literal) only limited analysis can be performed usually using MS Excel possibilities. This analysis usually incorporates creation of charts, histograms, paretos of defect etc. Not allowing to analyse non numerical data like area of the defect location, significance of the defect etc.

On top of this every production department requires a different point of view on this base of data and thus performs a different analysis. Quality requires analysis of NRFT, scraps etc. Production requires analysis of parts produced per hour/per shift/per day in relation to certain part, product group or even production location or supervisor, manufacturing engineering is interested into the data showing possible defects coming out of process or tooling etc. Thus every department has it's own data analysts (special function or joint function within a department), performing such analysis, usually occupying up to $20 \%$ of the working time of employee. This issue costs companies a lot of money in salaries, which could be invested to better usage of specialist's abilities on improvements within a process or product.

Cross communication between different production subgroups working than as a internal customer/supplier (e.g. one production line is supplying cut cloths to another one which is covering them) is than not possible as the data are not available in real time.

As well exchange of information and reaction possibilities between different departments is questionable.

\section{SOLUTION HIGHLIGHTS}

To cover all above mentioned requirements an robust solution is required with efficient data collection, business intelligence capabilities and analysis support required for fast decision making process speeding up reaction in case of nonconformity.

Important is to think about the architecture of the system and combine clientserver possibilities together with application support. So the data collection subsystem can be located on any IO device (portable device/laptop/fixed touch screen) any platform (windows, OSX, Android) to cover all requirements of various implementation possibilities.

Placement of the data acquisition device should be in the place where the data collection is taking place, meaning where is declaration of the conformity of the 
part. This is usually Final Control workstation, but can be any other workstation depending of the flowchart of every particular process (Quality Wall, Warehouse, Product Audit location etc.). For the sake of efficiency of the data acquisition all ergonomic standards have to be respected taking into account height and distance from operator performing data entry up to the light conditions and visibility of the information on the screen.

To improve the work content related to this data entry it is essential to define the IO device allowing minimum movements needed to be done by operator. The best solution nowadays is the touch screen possibilities where the IO device is incorporating displaying of information with input required from operator.

During the application design principles of effective allocation of screen content should be analysed in detail. Meaning, showing the important information very obvious way and information of low priority requiring lower attention of operator with less expressive design.

As already mentioned manual entry of data is mostly dependent on the operator concentration and discipline. To allow keeping this discipline is required to decrease the amount of the operations which operator has to perform to minimum. Expected value should be around 5 operations, acceptable 10. In case of operations exceeding 20 separate actions have to be defined in order to deploy more data entry workstations with separate manpower. In order to speed up the data collection, possibility of incorporating the barcodes scanning all possible information out of the process or product is a best practice allowing to decrease need of operator input (e.g. scanning of reference of the part to the system directly without typing information directly, scanning of fault code, number of operator etc.).

Whole system can be divided into 2 separate subsystems. First system solely for data acquisition allowing real time gathering of data with live connection to data servers (fix line or wireless) and second allowing data analysis, reporting, export of data to other systems like ERP etc. This solution will allow multiplication of data entry workstations connected to one data source allowing multiple entries to be done in the same time. As well robustness of the system will be ensured as system consists of 3 separate subsystems (servers/databases, data acquisition and data analysis) and in case of issue of one subsystem, another one can still work.

Analytical subsystem can be an application where access is allowed to all relevant parties (production, support functions, management etc.) with various levels of access. Using this principle all involved parties are working over one data source not using various data coming from different locations and systems. Important in this point is the accessibility of all the data in real time for all users to allow quick and efficient overview of the situation and define actions in case of deviations.

To speed up this process it is essential to have personalized overviews and reports already predefined in the analytical subsystem based on each function and/or department as every detail and point of view of separate function is 
different. Automatisation of creation of those reports can lead to system of notifications over email, phone, and intranet etc. decreasing needed reaction time. As well the platform on which analytical part will be run can vary from standard PC up to any mobile device like phone/tablet etc.

In case of special events or in case of significant deviations from standard it is worth to have the possibility to create on demand analysis and reports using simple user interface allowing various combinations of views on the base data allowing deeper analysis of the situation and current topic e.g. analysis of the defect coming from particular shift, particular time of production, particular operator etc.

Analysis and visualization of the data should be done in graphical form allowing to see the overview of actual situation as well with trends to allow anticipation of the future situation and take necessary actions in real time decreasing a risk of deviations in future. Concerning the defect visualization, not only standard tolls like ppm analysis, pareto etc. can be used but also possibilities like defect maps, comparative analysis etc. are useful.

\section{RESULTS AND DISCUSSIONS}

All of above mentioned requirements were deeply analysed within a cross sectional team consisting of management, quality, engineering and production functions allowing to create a common timeline for implementation of the system which has been called KONIS. Within this timeline a series of milestones has been defined allowing to control the implementation process and react in case of deviations to timeline.

Development of the system started in late 2012 and first pilot version consisting of 1 workstation with touch screen monitor has been implemented on the production line in 1st quarter 2013.

After pilot phase, spreading of fine-tuned system proceeded. Nowadays KONIS system is applied on 36 workstations covering all production lines in the company.

System allowed immediate saving of approximately 980 working hours for data analysis monthly performed by engineers, automatisation of data entry saved approximately 20 seconds per part control resulting in immediate saving of more than 1600 hours of operator time within a month what in the same time improved as well efficiency of the control. The most important outcome of the system implementation was increased transparency over the data collection and immediate overview of actual plant performance allowing quick and efficient actioning in case of deviations.

Inputs from KONIS are now widely used during problem solving processes allowing to cut the ratio of non conforming products by $75 \%$ within half a year and becoming a benchmark solution within Faurecia group. 
Major advantages of such system are:

- creation of the real time overview about the current situation on all production facilities/lines from the point of view of produced quantities, scraps or non-conformities and outlined parts flow;

- decreasing reaction time to non-conformity to minimum;

- anticipation of possible non-conformity based on trends and historical overview compared with actual situation;

- decrease of the headcount needed for data analysis and reporting;

- decreasing "data noise" coming from the manual entry of data;

- improving of customer perception of the company;

- detailed and efficient base for problem solving process.

Major challenges

- adoption of such system by all involved parties understanding its advantages;

- detailed and continuous trainings in production to promote and let understand the system;

- cross connection of the system with ERP/MRP system within a production entity;

- efficient IT support related to functionalities of whole system;

- further development of the system to become valuable business intelligence tool within a company.

Further research and development of the system would be possible in close integration of the system itself into the ERP/MRP system and as well creating of business intelligence possibilities with further support of decision making process within an organization.

\section{ACKNOWLEDGEMENT}

This paper is handled as part of KEGA 009TnUAD-4/2011 Creative Laboratory Engineering Education at Technical Faculties „CRELABTE“ supported by Ministry of Education, Science, Research and Sport of the Slovak Republic.

\section{REFERENCES}

CADCAM Integration, 2013. SuiteMDI. A Manual Data Inputing Application with Support for SuiteMonitoring. [Online] Available at: <http://www.suitefactory.com/htmlbrochures/suitemdi.htm> [Accessed. 15 November 2013]. 
Pegasie Technologies, 2013. Intelligent data entry automation. Available at: <http://www.pegasie.com/intelligent_data_entry_automation_(idea).php> [Accessed 15 October 2013].

Points North, 2013. How to Better Monitor Work Floor Performance, Streamline Tracking Processes and Obtain Accurate, Up-to-the-minute Data. Available at: $\langle$ http://www.points-north.com/whitepapers/lstouchdata.pdf> [Accessed 2 September 2013].

Production Process, 2013. ProductionACE Reports: Standard, Custom and Export to Excel and Other Applications. Available at: $<$ http://productionprocess.com/> [Accessed 10 November 2013].

Robbins, K., 2013. Problems With the Manual Entry of Data. Available at: <http://www.ehow.com/print/info_8101077_problems-manual-entry-data.html> [Accessed 10 October 2013].

Warren Wolfe, 2010. How to Better Monitor Work Floor Performance, Streamline Tracking Processes and Obtain Accurate, Up-to-the-minute Data Available at: 〈http://www.points-north.com/whitepapers/lstouchdata.pdf >.

Weir, C.R., Hurdle, J.F., Felgar, M.A., Hoffman, J.M., Roth, B., et al., 2003. Direct text entry in electronic progress notes. An evaluation of input errors. Methods Inf Med 42 (1/2003), pp. 61-67, Available at: <http://square.umin.ac.jp/DMIESemi/y2003/20040216/03010061.pdf〉.

Wahi M.M., Parks D.V., Skeate R.C., Goldin S.B., 2008. Reducing errors from the electronic transcription of data collected on paper forms: a research data case study. J Am Med Inform Assoc. 2008 May-Jun; 15(3), pp. 386-389. doi: $10.1197 /$ jamia.M2381

Rieder HL, Lauritsen JM, 2011. Quality assurance of data: ensuring that numbers reflect operational definitions and contain real measurements. Int J Tuberc Lung Dis, 2011 Mar; 15(3) pp 296-304.

MacKenzie, I. S., \& Soukoreff, R. W. (2002). Text entry for mobile computing: Models and methods, theory and practice. Human-Computer Interaction, 17, pp. 147-198. [Online] Available at: 〈http://www.yorku.ca/mack/hci3.html> [Accessed 1212 2013].

\section{ABOUT THE AUTHOR}

Lubomir Lengyel is acting as a quality manager in Faurecia Leather Kosice, member of Faurecia Group, leading supplier of interior systems in automotive industry, e-mail:lubomir.lengyel@faurecia.com, VSB - Technical University of Ostrava Ph.D. postgraduate student, e-mail: lubomir.lengyel@vsb.cz. 